\title{
LES ENTREPRISES DE PÊCHE ARTISANALE : ESSAIS DE TYPOLOGIE
}

\author{
Joseph CATANZANO*, B. GILLY* et F. LANTZ*
}

\section{Résumé :}

Cet article présente les résultats des tentatives d'élaboration d'une typologie des entreprises de pêche artisanale française. L'originalité de ces essais tient au rôle majeur qui est accordé aux variables économiques et ce en vue d'analyser ces flottilles au regard de données comptables sur un échantillon national puis régional. L'application théorique des ratios économiques et financiers au secteur des pêches artisanales permet d'en dresser un diagnostic plutôt sombre. La sélection d'une zone géographique et l'approche menée en terme d'analyse factorielle des correspondances multiples permettent de croiser les caractéristiques d'exploitation des bateaux avec les critéres de rentabilité précédemment évoqués et ce afin d'étudier leurs liens.

\section{Summary :}

\section{THE ARTISANAL FISHING ENTERPRISES : SOME ATTEMPTS OF TYPOLOGY}

This paper presents some attempts for a French artisanal fishing enterprises typology. The originality of these attempts arises from the lead given to economic variables in order to analyze the fishing fleet using accountable datas both at national then regional level. The theoric use of economic and financial ratios (added value, solvency and financial autonomy) leads to the presentation of the artisanal fleet economic situation. The choice of a geographic area and the use of multiple-correspondance-analysis permits a cross analysis of fisheries features and return criteria.

La modification de la structure de production du secteur des pêches artisanales françaises, amorcée dès la fin de la seconde guerre mondiale, s'est accélérée à la fin des années soixante. Un processus d'intensification du capital est venu modifier le poids respectif des différents facteurs de production utilisés. Le nombre de navires ainsi que le nombre d'emplois diminuent. La puissance, la taille et l'équipement des bateaux augmentent dans le même temps. Les quantités débarquées stagnent même si une modification qualitative des apports et surtout une demande croissante permettent une augmentation en francs constants de la valeur des débarquements (Meuriot, 1987). Il est vraisemblable que l'évolution technique des navires a été largement favorisée par l'effet des modifications successives des conditions de financement des unités de production (le Crédit Maritime prend son essor au début des années soixante) et par l'évolution positive de la masse des subventions allouées à cette composante des pêches maritimes.

A cet égard, les années soixante-dix et la période du VIIème Plan sont significatives d'une volonté étatique d'accompagner, voire d'accélérer cette mutation. Dès lors que cet accroissement du capital utilisé s'inscrit sur les dernières décennies, indépendamment semble-t-il des évaluations de l'état des ressources et de l'estimation du niveau d'exploitation de celle-ci, des déséquilibres sont apparus, d'ordre social, économique et biologique.

Lorsque l'on connaît les critères d'intervention de l'Etat, des Régions, de la Communauté Européenne en matière de subvention à l'investissement et les règles de financement appliquées, il paraît important de vérifier si ces modalités de traitement des différentes composantes de la flottille artisanale ont un fondement économique autre que des caractéristiques de taille (volume du chiffre d'affaires, immobilisations...). Ainsi on peut s'interroger pour savoir si les critères de longueur et autres caractéristiques techniques, dont on connaît le rôle dans la détermination des modes de financement ou les niveaux de subvention accessibles, sont significatifs de différentes formes d'entreprises. Pour cela, dans un premier temps, on a cherché à mettre en évidence une possible classification économique des entreprises à partir des seules données comptables. Dans une seconde étape, l'objectif sera de croiser cette information économique avec des critères techniques sur un échantillon particulier améliorant ainsi la qualité explicative de l'analyse.

\section{UNE BASE DE DONNÉES UNIQUE POUR UN SEC- TEUR MARQUÉ DE FORTES SPÉCIFICITÉS}

Le secteur de la pêche artisanale présente des caractéristiques économiques originales par rapport aux autres secteurs. 11 convient de souligner en particulier l'importance du capital investi, alors qu'il s'agit surtout d'entreprises individuelles. Le coût actuel de chalutiers neufs de 20 à 24 mètres (d'une puissance motrice d'environ 400-500 CV) se situe entre 5 et 8 millions de FF. La comparaison avec les données de la flottille industrielle (CACEPAANIA, 1983), montre qu'en terme de ratios économiques de base ces composantes des pêches artisanale et indus-

- Département Stratégie de Développement et Aménagement.

Institut Français de Recherche pour l'Exploitation de la Mer (IFREMER) 
trielle ne sont pas si éloignées (tableau 1).

Tableau 1. - Comparaison des flottilles artisanale et industrielle (1983)

Flottille artisanale (echantillon CGPA) 0.036 0.318

Résultat net/CA

Rémunérations/CA

Investissement/CA

(en notant CA le chiffre d'affaires)

Des évolutions naturelles ou induites des niveaux de production peuvent rompre à tout moment et sur n'importe quelle période les tendances évolutives en œuvre. Lors de ruptures accidentelles, des effets de marché peuvent venir compenser totalement ou partiellement ces fluctuations mais rien n'est a priori systématique ou du moins généralisable à toutes les espèces pêchées.

Les données utilisées proviennent du Centre de Gestion de la Pêche Artisanale. A ce jour, il s'agit de la seule source d'informations capable de fournir sur un échantillon national des données homogènes dans leur traitement et actualisées chaque année depuis 1983. Autant dire que malgré les limites de ces documents comptables, sur lesquelles on reviendra par la suite, rien de plus exhaustif n'existe aujourd'hui concernant cette composante artisanale des pêches maritimes françaises. Les dossiers disponibles, regroupés au CGPA, se présentent en fait sous la forme de synthèses des liasses fiscales. Les adhérents sont tous nécessairement imposés selon le régime réel (normal ou simplifié), de plein droit ou sur option (1). Les postes

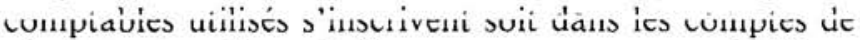
résultats, le tableau de financement, ou encore le bilan. Quelques données complémentaires relatives aux effectifs embarqués, aux caractéristiques techniques des bateaux, au nombre de jours d'activité sont aussi disponibles.

Pour l'année 1986, les services du Ministère de la Mer recensent 1900 navires artisans correspondant aux caractéristiques de l'échantillon. L'échantillon global dont on dispose (619 unités), est à l'évidence constitué par des bateaux ayant en général un chiffre d'affaires au moins supérieur à $500 \mathrm{KF}$, ce qui exclut de facto les petites unités de production. Ce biais n'est pas sans conséquence sur la portée des résultats et des conclusions présentées ici. Par ailleurs, I'adhésion à un groupement de gestion des entreprises ayant un chiffre d'affaires voisin de $500 \mathrm{KF}$ est dans une large mesure conditionnée par l'appréciation des avantages liés à cette adhésion : possibilités d'attribution d'une prime lors de la construction d'une unité de pêche, abattement fiscal lié à l'adhésion à un centre de gestion. Du point de vue fiscal, l'importance des bénéfices déclarés est telle que l'on peut supposer qu'à diverses étapes des comptes de résultats et du bilan, des valeurs puissent être augmentées d'autant plus facilement qu'elles correspondent à des postes quasi-fictifs (amortissement par exemple).

\footnotetext{
1. Différents régimes d'imposition :

- au forfait si le chiffre d'affaires est inférieur ou égal à $500000 \mathrm{Frs}$. Les copropriètés en sont exclues.

- au réel simplifié si le chiffre d'affaires est compris entre 500000 et 3000000

Frs ou sur option.

- au réel normal si le chiffre d'affaires est strictement supérieur à 3000000 Frs ou su option.

- Par rapport au réel normal, le réel simplifié permet un allégement des formalités déclaratives et une simplification des documents transmis aux Impóts bilan abrégé, compte de résultats et annexes simplifièes... Les règies d'assiette
}

\section{L'ANALYSE DES ENTREPRISES A L'AIDE DE} RATIOS

Appliqués à l'échantillon de l'année 1986 (619 bateaux), six ratios permettent d'obtenir une première distribution des bateaux selon divers critères économiques. Les six ratios utilisés se regroupent de la façon suivante :

- Indicateurs d'autonomie financière :

RA1 = capitaux propres / (emprunts + passif exigible)

RA2 = capitaux propres $/$ emprunts

- Indicateurs de solvabilité :

RA3 = disponibilités $/$ passif exigible

RA4 = actif circulant $/$ passif exigible

- Indicateur de résultat d'exploitation :

RA5 = valeur ajoutée / chiffre d'affaires

- Indicateur d'engagement financier :

RA6 = frais financiers / chiffre d'affaires

Les deux premières valeurs (RA1 et RA2) nous renseignent à partir d'éléments de structure du bilan, sur l'équilibre financier d'une entreprise au regard de ses engagements à long ou moyen terme (emprunts) et de ses capitaux propres. On considère généralement, dans les secteurs d'activités autres que la pêche, que l'ensemble des capitaux propres doit gager le montant des emprunts. L'endettement à moyen et long termes ne saurait excéder la valeur de ces capitaux propres si l'entreprise veut conserver son independance, son autonomie de decision et d'action. II est tout aussi important de préciser qu'à l'inverse, des valeurs trop élevées de ces ratios peuvent signifier que l'on est dans le cas d'entreprises dont le rythme d'investissement est faible et qui peuvent donc s'avérer peu dynamiques.

Les indicateurs de solvabilité concernent eux aussi des éléments du bilan relatifs aux engagements à court terme. Ils se différencient l'un de l'autre uniquement par référence au temps de mise à disposition qu'ils nécessitent. L'un est calculé sur la base des liquidités immédiatement disponibles (RA3), l'autre sur la base d'éléments récupérables à court terme (RA4). Le cinquième ratio (RA5), relatif aux résultats, mesure la part de richesse créée dans le volume d'activité.

S'agissant de l'engagement financier (RA6), on dispose là d'un élément intermédiaire d'appréciation à la fois du niveau d'endettement (long et moyen termes) mais aussi un indicateur pesant à court terme sur les résultats économiques de l'entreprise. Un seuil d'acceptabilité fixé à $3 \%$ est retenu pour les autres secteurs, certains pays ayant même opté pour une réglementation à partir de ce pourcentage de chiffre d'affaires.

(base de calcul de l'impôt) et les déclarations de résultats sont identiques. - L'abattement fiscal lié à l'adhèsion au CGPA oblige aux conditions suivantes - être assujetti à l'impôt sur le revenu

être placé sour un régime réel d'imposition :

avoir adhéré au centre de gestion pendant toute la durèe de l'exercice. L'abattement s'exerce sur le bénéfice fiscal de l'entreprise, déclaré par l'artisan dans la catégorie des "Bénéfices industriels et Commerciaux". Deux taux distincts (20\% et $10 \%$ ) s'appliquent sur des fourchettes différentes avec détermination d'un abattement maximun. 
Pour repère, en 1983 l'industrie automobile française présentait un taux d'endettement moyen de l'ordre de $36 \%$ pour un taux de valeur ajoutée voisin de $33 \%$ (Devilliers, 1985). Pour l'artisanat et pour la même année, les taux étaient respectivement de $35 \%$ (entreprises employant entre 3 et 10 salariés) et $30 \%$ (Thibaud, 1988 et TEF, 1987.)

Graphique 1. - Distribution des effectifs du CGPA en 1986 selon les valeurs des différents ratios
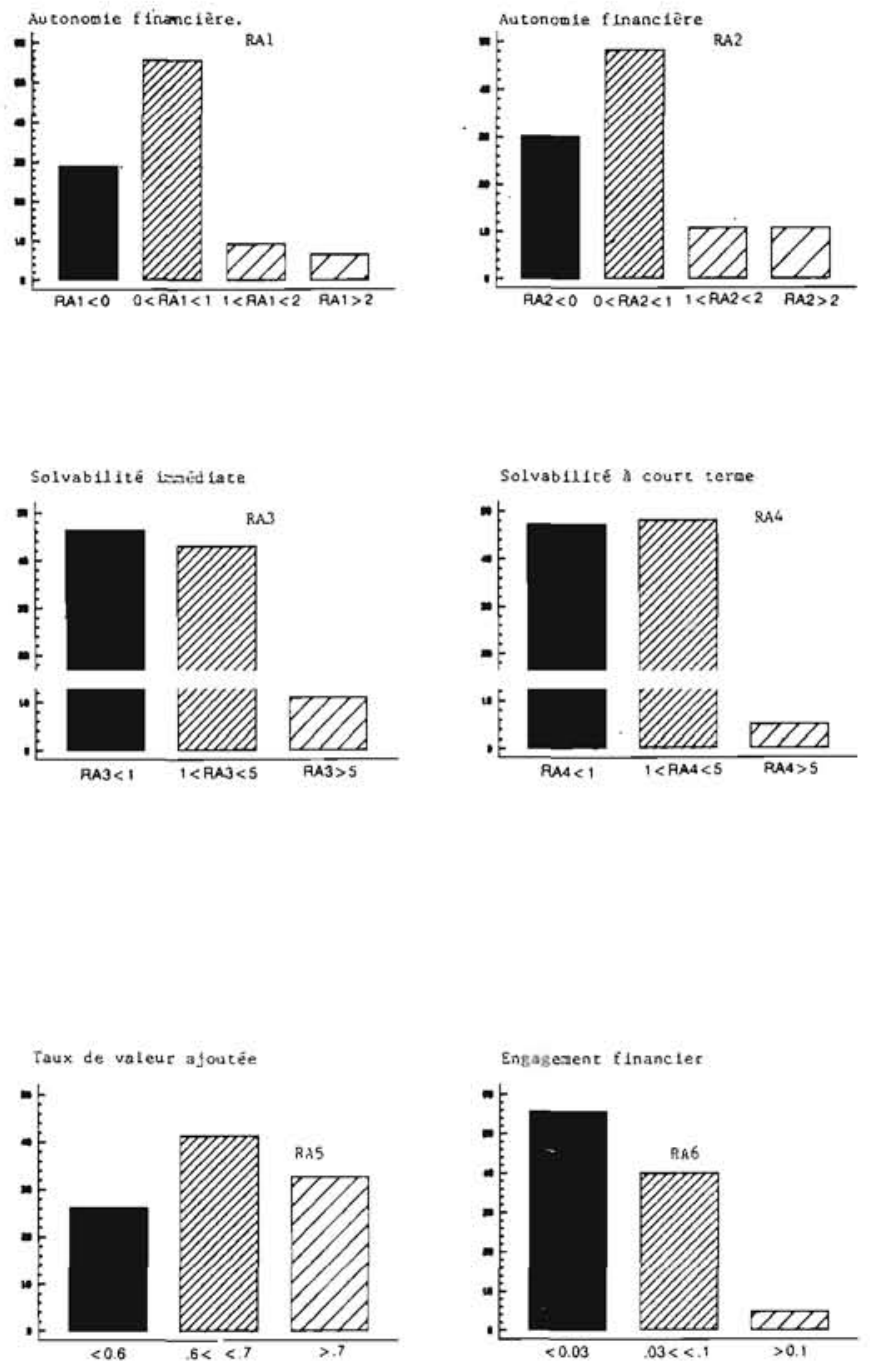

A l'analyse des ratios (graphique 1), on constate une absence d'autonomie financière (pour près de $80 \%$ des unités en 1986) expliquée par des capitaux négatifs (pour plus d'un tiers de celles-ci), conséquence directe d'un résultat d'exercice lui aussi négatif ou de forts prélèvements réalisés par l'entrepreneur ; une absence également de solvabilité à court terme pratiquement pour une entreprise sur deux ainsi qu'un niveau de frais financiers important pour une proportion similaire d'entreprises.

Globalement ce sont des indicateurs financiers qui révèlent en théorie des gestions (à long terme et à court terme) plutôt problématiques. En effectuant plusieurs croisements (tableau 2) on arrive à extraire parmi les bateaux n'ayant pas d'autonomie financière (capitaux propres < emprunts) un groupe important qui se caractérise aussi par un taux de valeur ajoutée des plus élevés (près de $58 \%$ de l'échantillon total), puis, bon nombre d'entreprises n'ayant pas de solvabilité immédiate ou à court terme (près de la moitié de l'échantillon) et enfin un ensemble comprenant la quasi totalité des entreprises devant couvrir des frais financiers importants par rapport au chiffre d'affaires (près de $44 \%$ de l'échantillon 1986). Comment se répartissent ces indicateurs sur l'ensemble de la population étudiée?

Si on tient compte de ces critères pour les unités ayant un taux de valeur ajoutée supérieur à 0,6 , ce sont $18 \%$ des entreprises que l'on recense en 1986. Si on se libère tour à tour de l'une ou l'autre des contraintes de classification précédentes, on arrive aux pourcentages indiqués ci-dessous :

Critère $\mathrm{A}=$ pas d'autonomie financière

$78,4 \%$

Critère $\mathrm{B}=$ pas de solvabilité immédiate $57,7 \%$

Critère $\mathrm{C}=$ taux valeur ajoutée $>0,6$ $73,7 \%$

Critère $D=$ fort taux de frais financiers $44,3 \%$

Critères A-B-C-D $17,9 \%$

Critères A-B-C $28,8 \%$

Critères A-B-D

$28,8 \%$

En rejetant toute condition sur le taux de valeur ajoutée, c'est-à-dire en privilégiant les éléments de structure du bilan, l'effectif d'entreprises en situation critique représente encore un pourcentage non négligeable $(29 \%)$.

Tableau 2. - Analyse des ratios financiers

\begin{tabular}{|c|c|c|c|c|}
\hline Pour RA1: & $\leqslant 0$ & $0<. \leqslant 1$ & $1<\leqslant 2$ & $>2$ \\
\hline$R A 3 \leqslant 1$ & 24.6 & 29.0 & 2.4 & 1.5 \\
\hline$R A 3>1$ & 4.2 & 26.5 & 6.8 & 5.0 \\
\hline$R A 4 \leqslant 1$ & 22.6 & 22.5 & 1.3 & 0.7 \\
\hline RA $4>1$ & 6.2 & 33.0 & 7.9 & 5.8 \\
\hline$R A 6 \leqslant 0.6$ & 12.6 & 10.2 & 1.6 & 1.6 \\
\hline $0.6<$ RA $6 \leqslant 0.7$ & 10.2 & 25.4 & 3.2 & 2.4 \\
\hline RA $6>0.7$ & 6.0 & 19.9 & 4.4 & 2.5 \\
\hline$R A 8 \leqslant 0.03$ & 12.3 & 28.9 & 7.9 & 6.5 \\
\hline RA $8>0.03$ & 16.5 & 26.6 & 1.3 & \\
\hline TOTAUX & 28.1 & 55.5 & 9.2 & 6.5 \\
\hline \multicolumn{5}{|l|}{ RA2 : } \\
\hline$\leqslant 0$ & $0<\leqslant 1$ & $1<\leqslant 2$ & $>2$ & TOTAUX \\
\hline 25.6 & 24.4 & 4.5 & 3.1 & 57.5 \\
\hline 4.5 & 23.8 & 6.3 & 7.7 & 42.5 \\
\hline 23.5 & 18.7 & 3.1 & 1.7 & 47.1 \\
\hline 6.6 & 29.6 & 7.7 & 9.1 & 52.9 \\
\hline 13.2 & 9.5 & 1.2 & 2.4 & 26.0 \\
\hline 10.7 & 22.0 & 5.0 & 3.3 & 41.0 \\
\hline 6.2 & 16.8 & 4.6 & 5.1 & 32.8 \\
\hline 12.5 & 21.1 & 8.9 & 10.6 & 5.6 \\
\hline 17.6 & 27.2 & 1.9 & 0.2 & 42.0 \\
\hline \multicolumn{5}{|l|}{ TOTAUX } \\
\hline 30.1 & 48.3 & 10.8 & 10.8 & 100.0 \\
\hline $\begin{array}{l}\text { RA1 = capitau } \\
\text { RA2 = capitau } \\
\text { RA3 = disponit } \\
\text { RA4 = actif cir } \\
\text { RA5 = valeur } \\
\text { RA6 = frais fir }\end{array}$ & $\begin{array}{l}\text { opres / e } \\
\text { opres / e } \\
\text { és / passi } \\
\text { ant / pas } \\
\text { itee / chi } \\
\text { ciers / chi }\end{array}$ & $\begin{array}{l}\text { ts + pass } \\
\text { ts à moye } \\
\text { ble } \\
\text { ible } \\
\text { affaires } \\
\text { affaires }\end{array}$ & $\begin{array}{l}\text { gible } \\
\text { ne }\end{array}$ & \\
\hline
\end{tabular}

En conclusion de cette analyse conduite en termes de ratios, on peut proposer une interprétation des caractères dominants en rappelant au préalable que les résultats 
seront à recadrer par rapport aux caractéristiques financières propres au secteur ou en référence à d'autres composantes de celui-ci (par exemple les flottilles industrielles). Cette composante CGPA des pêches artisanales témoigne d'une dépendance et d'un endettement importants. Cette situation pourrait se comprendre d'autant mieux si on démontrait le rôle incitateur souvent supposé des différentes aides accordées au secteur (subventions directes et bonifications). Cet encadrement financier justifierait alors ce comportement particulier des investisseurs intégrant implicitement dans le mode de gestion la pérennisation des aides. Le jugement que l'on énonce, si on tient compte d'une analyse traditionnelle des ratios, perd forcément de sa signification dès lors que l'on prend en compte les règles d'intervention qui viennent corriger les indicateurs obtenus.

Sans référence à l'environnement financier spécifique, le secteur apparaît fragile, puisque l'autonomie financière y est rare, la solvabilité immédiate plutôt absente et la charge d'intérêt financier non négligeable. Rappelons ici les $29 \%$ d'unités qui réunissent ces trois caractères.

Graphique 2. - Projection sur le plan principal 1-2.

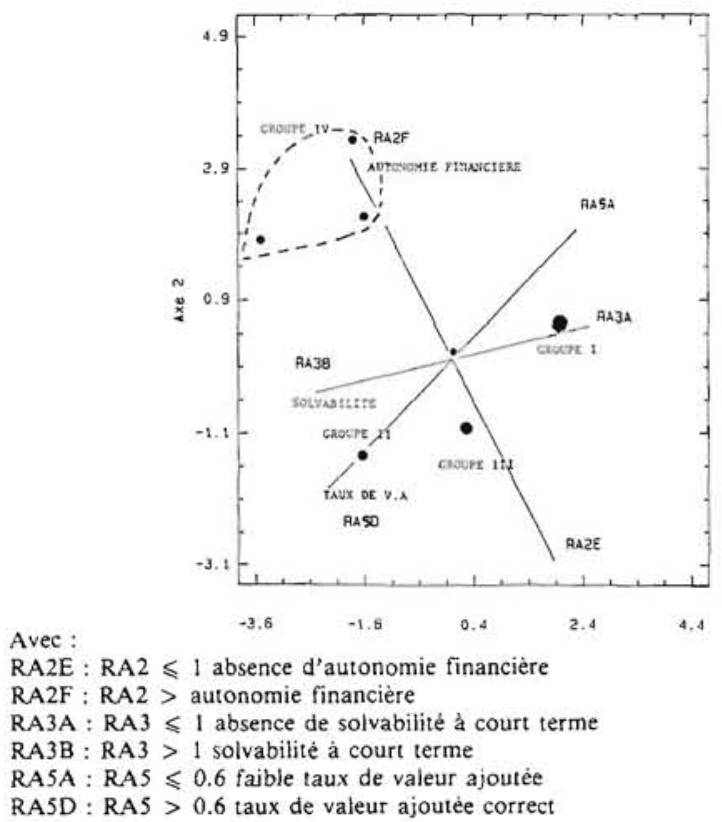

\section{CLASSIFICATION PAR L'ANALYSE DES CORRES- PONDANCES MULTIPLES}

L'analyse du tableau donnant les valeurs des ratios pour l'échantillon des entreprises de pêche étudiées n'a pas pu être menée à partir d'une analyse en composantes principales. L'évolution des ratios n'étant pas linéaire, les valeurs obtenues pour ceux-ci ont été réparties selon différentes classes pour effectuer une analyse factorielle des correspondances multiples (AFCM).

La première analyse que nous avons réalisée a porté sur quatre cents entreprises et trois ratios. Les trois caractères - ratios RA2 (autonomie financière), RA3 (solvabilité) et RA5 (taux de valeur ajoutée) - ont été chacun découpés en deux modalités (à partir des valeurs seuil définies précédemment).

Les trois axes principaux expliquent les pourcentages d'inertie suivants : $54,36 \%, 27,88 \%, 17,75 \%$. La repré sentation graphique de ce plan fait apparaître sur le premier axe l'opposition entre les modalités correspondant à de bonnes valeurs (RA2F, RA3B et RA5D) ou à de mauvaises valeurs de ratios (RA2E, RA3A et RA5A). Le deuxième axe est marqué par l'opposition entre les bonnes et les mauvaises valeurs du ratio d'autonomie financière (caractère RA2). La projection des individus sur le plan principal 1-2 (graphique 2) permet de distinguer trois groupes, dominants en poids, caractérisés par :

- Groupe I : pas de solvabilité, pas d'autonomie financière et faible taux de valeur ajoutée.

- Groupe II : bon taux de valeur ajoutée mais pas d'autonomie financière malgré une solvabilité moyenne.

- Groupe III : autonomie financière défavorable, solvabilité insignifiante et taux de valeur ajoutée moyen.

Ces trois groupes représentent près de $80 \%$ de l'échantillon. Un dernier ensemble (Groupe IV) de faible taille $(13 \%)$ réunit les unités aux indicateurs financiers et économiques plutôt favorables. Le reste de l'échantillon ne permet pas de dégager un archétype dominant au sens des trois ratios.

\section{VERS UNE AMÉLIORATION DE LA CLASSIFICA- TION DES ENTREPRISES}

La première tentative de classification des entreprises de pêche artisanale en fonction des critères économiques reste encore limitée, en raison de l'extrême hétérogénéité des données, tant en valeur absolue qu'en valeur relative. Il pouvait dont paraître opportun d'approfondir les analyses à partir d'une zone géographique particulière dont les caractéristiques de l'activité de pêche sont connues. La zone du Finistère a été retenue en raison, à la fois de l'existence de travaux antérieurs réalisés sur cette région (Charuau et al., 1986 ; Catanzano, 1987), de l'importance de la pêche artisanale finistérienne au regard de la pêche française ( $24 \%$ en quantité et $22 \%$ en valeur des débarquements en 1985), de la bonne représentation des entreprises de cette région dans la population du CGPA et enfin de l'existence de plusieurs types de pêche.

\section{Les groupes stratégiques}

L'AFCM a porté sur les caractéristiques structurelles (techniques) des entreprises de pêche et leurs paramètres économiques en matière d'exploitation, de rentabilité et d'investissement. Huit variables ont été ainsi retenues : le chiffre d'affaires, le taux de valeur ajoutée, le taux d'excédent brut d'exploitation, un indicateur d'intensité capitalistique, le niveau des capitaux propres, le niveau des immobilisations, un indicateur d'investissement et un ratio de capacité d'emprunt.

Le chiffre d'affaires a été choisi pour rendre compte de la dimension de l'activité économique de l'entreprise et a été réparti entre trois modalités autour des valeurs seuil 1,5 et $3 \mathrm{MF}$ (modalités PAU, MED et RIC). De même, le niveau des immobilisations, suivant qu'il est plus petit ou plus grand que 1 MF (modalités IM1 ou IM2), a été utilisé pour caractériser l'importance de l'entreprise.

Deux ratios ont été retenus pour décrire la richesse créée (taux de valeur ajoutée) ainsi que le degré de rentabilité (taux d'excédent brut d'exploitation). Le taux de valeur ajoutée a été séparé suivant deux classes (VA1 et VA2) suivant qu'il est inférieur ou supérieur à $60 \%$. Pour le second ratio, trois modalités (TX1, TX2 et TX3) ont été consti- 
tuées à partir des valeurs seuil $5 \%$ et $15 \%$. L'utilisation simultanée des deux ratios dans l'analyse permet de prendre en compte le mode de répartition particulier dans la pêche artisanale (rémunération à la part) qui est proportionnelle à la recette courante nette.

Deux variables décrivent l'importance relative du capital et la dynamique de l'investissement. L'indicateur d'intensité capitalistique est le rapport de l'investissement initial sur le chiffre d'affaires : il a été réparti entre trois modalités (K1, K2 et $\mathrm{K} 3$ ) à partir des valeurs seuil $50 \%$ et $100 \%$. La dynamique de l'investissement sur l'année 1985-1986 a été repérée par une variable binaire opposant l'investissement à son absence (modalités OUI et NON).

Enfin, deux variables caractérisent la situation financière des entreprises de pêche. Le niveau des capitaux propres a permis de définir deux classes suivant que celui-ci est négatif ou non (modalités KP1 et KP2). Le degré d'autonomie financière (ratio RAl défini précédemment) a été séparé en trois modalités (MP0, MP1 et MP2) suivant les valeurs seuil 0 et 1 .

Afin de mettre en évidence l'influence de certains facteurs extérieurs tels que les caractéristiques structurelles, les variables techniques ont été considérées comme variables supplémentaires. Dans la région étudiée, à chaque métier retenu correspond une durée de temps de pêche (marée) bien distinct. Ainsi, suivant la durée de la marée, une variable "métier pratiqué" a été définie en quatre modalités : INC (inconnu) pour les durées non précisées, PEF (pêche fraîche) pour les petites unités réalisant des marées de moins de trois jours, POI (poissonniers) lorsque les marées durent entre 3 et 10 jours, LAN (langoustiniers) pour les chalutiers hauturiers qui effectuent des marées de plus de 10 jours.

Les unités de pêche ont été distinguées suivant leur puissance motrice en trois modalités $(200,300$ et 450$)$ à partir des valeurs seuil 200 et $400 \mathrm{CV}$. Enfin, l'âge des navires a permis de répartir les bateaux en deux modalités (YOU et OLD) suivant que ceux-ci avaient moins ou plus de 8 ans.

La mise en évidence de l'importance décroissante des axes étant parfois délicate sur une AFCM, nous avons préféré calculer les taux d'inertie modifiés $\left(\mathrm{tm}_{1}\right)$ correspondant à un tableau de Burt modifié (Benzécri et al., 1980) (tableau 3). Ceux-ci mettent ainsi en évidence l'importance des deux premiers axes qui expliquent à eux seuls $93,5 \%$ de l'inertie du nuage.

\begin{tabular}{ccccc}
$\begin{array}{c}\text { Tableau } \\
\text { Rang }\end{array}$ & \multicolumn{4}{c}{ Valeurs propres, taux d'inertie et taux modifiés. } \\
1 & $\mathrm{k}_{\mathrm{i}}$ & $\mathrm{t}_{\mathrm{i}}$ & $\mathrm{tm}_{\mathrm{i}}$ \\
2 & 0,32 & $22 \%$ & $75,5 \%$ \\
3 & 0,22 & $15 \%$ & 18 & $\%$ \\
4 & 0,17 & $11 \%$ & 4 & $\%$ \\
5 & 0,16 & $10 \%$ & $2,4 \%$ \\
\hline
\end{tabular}

L'axe 1 oppose les modalités K1 et IM1 aux modalités K3 et IM2. C'est un axe qui est déterminé par les paramètres financiers des entreprises et nous considérerons qu'il s'agit de l'axe des investissements. Le deuxième axe caractérise les modalités TX1 et VA1 d'une part, TX3 et VA2 d'autre part. Cet axe peut être considéré comme l'axe des résultats d'exploitation. L'axe 3 paraît fortement lié à la capacité ou à la propension des entreprises à investir sur leurs fonds propres. Nous le considérons comme l'axe de

\section{financement.}

Le plan principal 1-2 (graphique 3) fait apparaître, au niveau du premier quadrant, un groupe de navires qui associent à un fort niveau de chiffre d'affaires un niveau plutôt élevé d'investissements. A ce groupe de navires correspond essentiellement la modalité LAN. Cette relation, si on la rapproche des particularismes finistériens, confirme que les navires langoustiniers sont particulièrement efficaces en terme de résultat brut, mais constituent également les navires les plus portés vers l'investissement. En liaison avec l'axe 2, on met en évidence que les navires présentant un faible taux de valeur ajoutée sont également les moins rentables en terme d'excédent brut d'exploitation, ce qui suggère que les rémunérations sont préservées, même lorsque les conditions de rentabilité sont précaires.

Graphique 3. - Projection des caractères sur le plan 1 - 2.

(le nom des variables supplémentaires est noté en minuscule).

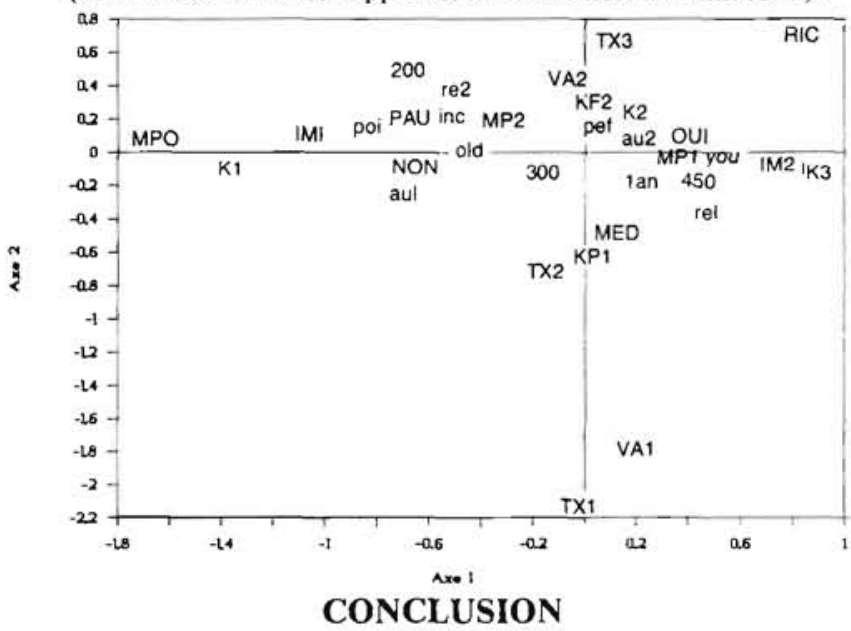

Les résultats obtenus montrent à l'évidence que l'image d'une pêche artisanale relativement homogène tant dans ses résultats que dans ses stratégies est désormais obsolète. Malgré les biais des échantillons utilisés, plusieurs points méritent d'être soulignés :

- les résultats économiques de cette composante des pêches artisanales sont globalement médiocres au regard des ratios couramment utilisés en analyse économique. Si les indicateurs d'exploitation et de rentabilité restent positifs, c'est surtout la situation financière du secteur qui est fragile avec un fort niveau d'endettement et une solvabilité réduite, conséquence de la modernisation de la flottille de pêche artisanale qui a permis, dans l'ensemble, d'améliorer la rentabilité des entreprises de pêche tout en les endettant lourdement ;

- l'analyse des ratios permet de modifier cette vue d'ensemble en distinguant quatre groupes d'entreprises, selon les niveaux de valeur ajoutée dégagée et de solvabilité, moins de un septième des individus de l'échantillon ayant une situation favorable au regard de l'ensemble de ces critères ;

- une typologie des entreprises réalisée sur la zone géographique du Finistère montre que les navires se distinguent non seulement par leur niveau de rentabilité mais aussi par leur dynamique d'investissements. Ces regroupements correspondent souvent à des caractéristiques de structures liées à l'âge du navire mais aussi à la stratégie de pêche. 
Les modèles bio-économiques traditionnellement utilisés font référence à des pêcheries simples (ou simplifiées par un jeu d'hypothèses adéquates), considérant le plus souvent l'exploitation d'une seule espèce par une flottille homogène. La construction de modèles permettant l'analyse de pêcheries composites (plusieurs types de navires en compétition pour la ressource) nécessite, d'une part que les navires soient regroupés en catégories homogènes au regard de leurs caractéristiques techniques (efficacité) et économiques (efficience) et, d'autre part, que soient connus leurs comportements d'investissements ou de désinvestissements (renouvellement des outils de production ou accroissement de leur efficacité).

De plus, ces essais de typologie apparaissent comme des passages obligés pour développer une analyse de l'adéquation des interventions du secteur public avec les résultats économiques des unités de production.

\section{BIBLIOGRAPHIE}

ANIA, CACEPA (1983). - Diagnostic sectoriel de l'armement hauturier. Paris, Document interne UAPF, 65 p.

BENZECRI J.P., BENZECRI F. (1986). - Pratique de l'analyse de données en économie. Paris, Dunod.

BENZECRI J.P., BASTIN C., BOURGARIT C., CAZES P. (1980). - Pratique de l'analyse des données. Abrégé Théorique et études de cas modèle - Tome 2. Paris, Dunod.

CATANZANO J., COCHET Y. (1987). - Investissement à la pêche en pays bigouden. IFREMER. Document de Travail 87/07. 27 pp.

CATANZANO J., GILLY B., LANTZ. F., DURAND M.H. (1988), - Analyse des résultats et des comportements économiques des entreprises de pêche artisanale. IFREMER. Rapport 88.015. 27 p.

CHARUAU A. (1986). - Etude d'une gestion optimale des pêcheries de langoustines et de poissons demersaux en mer celtique. CEEIFREMER, $149 \mathrm{p}$.
DEVILLIERS M. (1985). - Regard sur la situation des entreprises. Economie et Statistique, $\mathrm{n}^{\circ} 180$, Septembre.

MEURIOT E. (1985). - La flotte de pêche française. 1945-1983, Paris, IFREMER. Collection Ressources de la mer.

MEURIOT E., GILLY B. (1986). - Prix moyen mensuel des espèces démersales en France et à Boulogne sur Mer. Analyse économétrique de la période 1974-1983. IFREMER, Document de travail 86/12, $71 \mathrm{pp}$.

PEYRARD J. (1986). - Analyse financière des sociétés et groupes. Paris, Vuibert Ed. Coll. Vuibert Gestion.

REVUE FIDUCIAIRE (1986). - Le plan comptable général. La Revue Fiduciaire, Publications Fiduciaires.

SICARD C. (1987). - Pratique de la stratégie d'entreprise. Concepts opérationnels et procédures de succès. Paris, Hommes et Techniques Ed.

TEF. (1987). - Tableaux de l'Economie Française. Edition INSEE.

THIBAUD J. (1988). - Petites entreprises de l'artisanat, du commerce et des services. Collection INSEE, Série E $N^{\circ} 110,220 \mathrm{p}$. 